\title{
Probabilistic Management of Slope Disaster Detection Systems for Reduced Energy Consumption and its Convergence Property *
}

\author{
Kenji Hirata* Keigo Koizumi ${ }^{* *}$ João P. Hespanha ${ }^{* * *}$ \\ * Department of Mechanical Engineering, \\ Nagaoka University of Technology, Nagaoka 940 2188, Japan. \\ ** Department of Global Architecture, \\ Osaka University, 2-1 Yamadaoka, Suita 565 0871, Japan. \\ ${ }^{* * *}$ Department of Electrical and Computer Engineering, \\ University of California at Santa Barbara, \\ Santa Barbara, CA 93106 USA.
}

\begin{abstract}
Distributed networked sensor systems have a potential applicability for developing a slope disaster monitoring system. A low energy consumption property and an eventual long running life are an important issue in this type of outdoor application. This paper proposes a probabilistic strategy to turn on/off each sensor node that could reduce an entire amount of consuming energy over the network. Effectiveness of the proposed probability assignment strategy is evaluated through numerical experiments, and convergence property is also investigated.
\end{abstract}

Keywords: Sensor networks, Slope disaster monitoring, Energy consumption, Distributed decision making.

\section{INTRODUCTION}

Distributed networked sensing and control systems have a significant potential applicability in a broad range of areas Gharavi and Kumar (2003), Hespanha et al. (2007). This paper focuses on an application of the sensor network technology to a slope disaster detection problem, which have gained much interest in recent years Schwab et al. (2007), Mehta et al. (2007), Ramesh (2009), Terzis et al. (2006), Kim and Han (2008) and also in Japan as well Kitamura (2007), Chigira (2006), Ikegawa et al. (2009), Fukagawa et al. (2008), Nishiyama et al. (2008), since we have large highway and railway systems passing through a mountain area, and preventing a loss of life and property due to a slope failure is a significant issue.

The entire sensor network for the slope disaster detection consists of sensor nodes which are equipped with sensing and communication capabilities, where the sensor can measure potential indices of slope failure take place such as, for example, amount of deformation and soil-water. Corrected data will be sent to a base station through wireless interactions between the nodes, and each sensor node can also generate an alert signal to a facility manager in case of emergence. A distributed sensor network system, which can be used for the slope disaster detection, is required to have abilities to precisely measure the potential

\footnotetext{
* This work was supported in part by grant 24310139 and 23760386 from the Japan Society of Promotion of Science and by the Institute for Collaborative Biotechnologies through grant W911NF-09-D-0001 from the U.S. Army Research Office.
}

indices of slope failure and fast response, i.e., a small time delay in sending an alert message. As well as these fundamental requirements, a low energy consumption and an eventual long running life are an important property, since the network may be installed in a mountain area, and having a frequent maintenance such as changing power supply batteries is difficult. As an example, our field experiment study along the Japanese highway systems in Koizumi et al. (2012a) have reported that a specific sensor node has worked for four months with two C-size alkali butteries, and, in our current field experiment study in Koizumi et al. (2011), Koizumi et al. (2012b), a new hardware node device is running with lithium-ion batteries and is expected to work for more than two years. However, the running life is still not enough for a real practice, and we need to develop a management technique of each node device that can realize a reduced amount of consuming energy and consequently long running life.

Reducing the energy consumption of wireless communication devices can be investigated via efficient Media Access Control (MAC) and routing protocol designs Miller and Vaidya (2005). Another approach can be formulated as a sensor selection problem, where a state estimation problem under the communication bandwidth constraint due to a limited energy supply rate is considered. Efficient sensor selection strategies that minimize a certain cost function, usually related to the state estimation error, can be found in Jia et al. (2012), Mo et al. (2011), Shi et al. (2008), Gupta et al. (2006) and references therein. 
This paper investigates the problem of reducing the amount of consuming energy over the sensor network in a different and more straightforward setting. In the slope disaster detection problem, each node is equipped with a sensor that can detect a precise value of potential slope failure index, i.e., the sensor can measure a precise value of land deformation or an amount of soil-water at a specific location where the node is located. If a high possibility of the slope failure take place was detected, the node can immediately generate an alert signal. Because of this, we do not need to formulate a state estimation problem and can consider a straightforward procedure to reduce the amount of consuming energy such that turning off the sensing or communication capability of the specific node that is located at a point at which there is a low potential possibility of slope failures. More specifically, we propose a probabilistic turn on/off strategy of each sensor node, and if a sensor node detected a low slope failure index, then the node will turn on its sensing capability with a low probability in the subsequent time period. On the other hand, the sensing capability will be turned on with a high probability, if the node detected a high index of slope failure, and this high value of activation probability is also propagated through interactions between the nodes in order to awake the neighboring nodes and obtain a more precise data. Since a centralized operation for a large size network of sensors such as this application for slope disaster detection is not realistic, we propose a distributed probability assignments strategy, and each sensor node decides its subsequent action in distributed fashion utilizing corrected data from its neighboring sensors only. Effectiveness of the proposed strategy is evaluated through numerical experiments, and it shows that a resulting assigned activation probability can track a time evolution of unknown time-varying slope failure potential index. This paper also investigates the convergence property of the proposed algorithm. An experimental evaluation of the proposed algorithm using hardware devices have been reported in Hirata et al. (2013).

The reminder of this paper is organized as follows. The problem formulation is described in Section 2. In Section 3, we introduce the activation probability assignment problem. We propose specific probability assignment strategies in Section 4. In Sections 3 and 4, for the sake of simplicity, we consider the problem that involves activation or deactivation of the sensing capability only. An actual node device in our practice has sensing and communication capabilities. A generalized problem that involves activation or deactivation of both sensing and communication capabilities is considered in Section 5. Section 6 investigates the convergence property of the proposed algorithm. Concluding remarks are given in Section 7.

\section{PROBLEM FORMULATIONS}

We start by providing a basic notation, and the readers can refer to Fig. 1 . Let $S \subset \mathbb{R}^{2}$ be a bounded field of interest, where the sensor nodes are deployed. Let $\mathbb{R}^{+}$denote the set of non-negative real numbers, and consider an unknown time-varying potential function $\phi: \mathbb{R}^{+} \times S \rightarrow[0,1]$. One can regard $\phi$ as a function measuring the potential possibility that slope failures take place over the field. The larger the value $\phi(t, v)$, the higher probability of slope failure takes place at time $t \in \mathbb{R}^{+}$and location $v \in S$. We undensely deploy a finite number of sensor nodes over the field $S$ that having sensing and communication capabilities. A primal purpose of this sensor network is to detect a high value of the unknown potential function $\phi$ and make an alert signal. A possible rational strategy for this purpose is to turn on every sensor node at every time instance and correct the all possible data. However, this strategy consumes the largest amount of energy, and a long running life of each sensor node is a considerably important issue in this type of outdoor application. Our main interest in this paper is on an activation algorithm of sensing and communication capabilities of each sensor node, and we intend to reduce an amount of consuming energy as well as preventing a serious shortage of the amount of meaningful observed data.

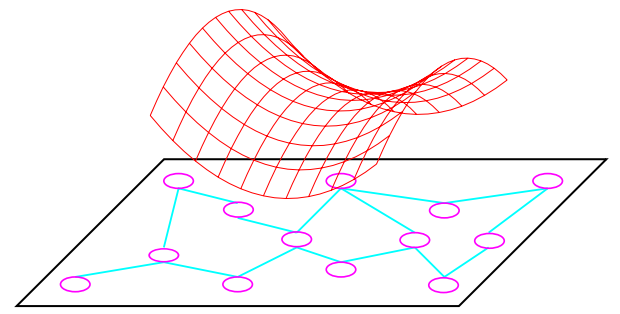

Fig. 1. A bounded rectangle region indicates the field $S$ of interest. A curved surface indicates the unknown time-varying potential function $\phi$, which is defined on the field $S$ and can be regraded as a function measuring the potential probability that slope failures take place over the field. The sensor nodes $P_{i}, i \in$ $N$ are located at the points indicated by circles, and a communication topology is described by the undirected edges between sensor nodes.

A group of sensors $P=\left\{P_{i} \mid i \in N\right\}, N=\{1, \ldots, n\}$ are deployed over the field $S$. Let $v_{i} \in S, i \in N$ denote the specific located point of $P_{i}$, and we also set $V=\left\{v_{i} \mid i \in\right.$ $N\}$. Let $\mathbb{Z}^{+}$denote the set of non-negative integers. At each time step $t \in \mathbb{Z}^{+}$, each sensor node is potentially allowed to interact with a group of other sensors, who are referred to as the neighbors of $P_{i}$, and we denote by $N_{i} \subset N$ the set of indices of the neighbors of $P_{i}$ and $\left|N_{i}\right|$ the number of elements in $N_{i}$. The interaction topology between sensor nodes is described in terms of undirected graph $G(V, E)$ with the set of nodes $V$ and the set of edges $E \subset V \times V$, where the set of indices $N_{i}$ satisfies $N_{i}=\left\{j \in N \mid\left(v_{i}, v_{j}\right) \in E\right\}$ for all $i \in N$. Since we consider undirected graph, $\left(v_{i}, v_{j}\right) \in E$ if and only if $\left(v_{j}, v_{i}\right) \in E$. We also assume $\left(v_{i}, v_{i}\right) \in E$ for all $i \in N$, i.e., the observed data by the node $P_{i}$ can be used by itself.

Example 1. Let us consider a simple rectangle field $S=$ $[1,100] \times[1,100]$. We deploy each sensor node $P_{i}$ with the located position $v_{i}=(4 h, 4 k) \in S, h, k=1,2, \ldots, 25$, $i=h+25(k-1)$.

We can consider an interaction topology such that $P_{i}$ can communicate to $P_{i}$ itself and the other sensor nodes located at left, right, top and bottom of it (see Fig. 2 ). $\square$

We suppose that each node $P_{i}$ is assigned an action set $A_{i}^{s}$, and, in our setting, the node can turn on its sensing capability or turn off it for saving the energy. We set $A_{i}^{s}=\{0,1\}$ for all $i \in N$, and the action $s_{i}(t)=1 \in A_{i}^{s}$ 


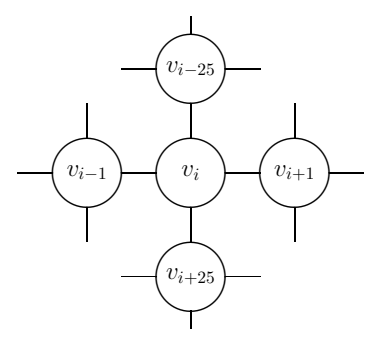

Fig. 2. A simple communication graph configuration.

implies that $P_{i}$ turns on its sensing capability at time $t \in \mathbb{Z}^{+}$and $s_{i}(t)=0$ implies turn off.

At every time step $t \in \mathbb{Z}^{+}$, each sensor node obtains an observed data $z_{i}(t)$ according to a selected action $s_{i}(t)$. We set the sensor output equation as

$$
z_{i}(t)=s_{i}(t) \times \phi\left(t, v_{i}\right) \quad t \in \mathbb{Z}^{+} \quad i \in N
$$

This setting means that the node $P_{i}$ with the active state $\left(s_{i}(t)=1\right)$ can get an exact value of the unknown potential function $\phi$ at time $t$ and location $v_{i}$ without any effect of observation noise. On the other hand, the output of the sensor node with the inactive state $\left(s_{i}(t)=0\right)$ is recognized as 0 .

In order to get the most precise information of the unknown potential function $\phi$, one can set $s_{i}(\cdot)=1$ for all $i \in N$, i.e., turn on every sensing capability at every time instance. However, this strategy consumes battery power even if the case $\phi(\cdot, \cdot) \approx 0$, i.e., turning on the sensing capability may have less meaning, since there is a very low possibility that a slope failure takes place. On the other hand, the case of $\phi(\cdot, \cdot) \approx 1$ implies a high possibility of slope failure, and we should turn on many sensors within a neighboring locations of this specific location and get precise data. We also could say that if the value of $\phi(\cdot, \cdot)$ is medium, it may be enough to turn on half the number of sensors and observe data with rough degree of accuracy.

We consider an activation strategy of the sensor nodes in the network that can handle a trade-off between the accuracy of observation and the amount of consuming energy. An important issue in this problem is that in a large scale networks of the sensors, i.e., the network has a huge number of sensor nodes, a centralized decision making is impossible. We propose a distributed decision making algorithm where the sensor node chooses its succeeding action by using only shared data with its neighbors.

\section{ACTIVATION PROBABILITY ASSIGNMENT}

We are interested in designing an activation strategy for each sensor node that can realize a low energy consumption. Our main idea is assigning an activation probability of the sensing capability of each sensor node. As we have discussed in the previous Section 2 , in case of $\phi(\cdot, \cdot) \approx 1$, we would like to turn on almost all sensing capabilities at this time instance and neighboring locations. On the other hand, if we had $\phi(\cdot, \cdot) \approx 0$, we need to turn off almost all sensing capabilities and consequently save energy.

Let $p_{i}^{s}(t) \in[0,1], t \in \mathbb{Z}^{+}, i \in N$ denote the activation probability of the node $P_{i}$, and the sensor node activates its sensing capability according to the assigned activation probability, i.e., $\operatorname{Pr}\left[s_{i}(t)=1\right] \approx p_{i}^{s}(t)$ (see algorithms in the following Tables 1 and 2 for details). In order to propose a specific activation probability assignment algorithm, we need to investigate a targeted activation probability assignment that can be regarded as an ideal solution to the problem. Let $q: \mathbb{R}^{+} \times S \rightarrow[0,1]$ and consider a monotonically non-decreasing function $\psi^{s}(q(t, v)) \in[0,1]$. The function $\psi^{s}$ is a design parameter in our problem, and the following two extreme examples represent a role of the function $\psi^{s}$.

Let us set $\psi^{s}(\cdot) \equiv 1$ and consider an activation probability assignment as $\psi_{i}^{s}(\cdot)=1 \approx p_{i}^{s}$. This means that turn on all the sensing capability at every time instance and every location, and this activation probability assignment strategy can get every possible data, but consumes the largest amount of energy. Another extreme choice is $\psi^{s}(\cdot) \equiv 0$, and an activation probability assignment $\psi_{i}^{s}(\cdot)=0 \approx p_{i}^{s}$ turns off every sensing capability and never consumes energy, but we can get no observed data. Although these two extreme examples are useless in a practical sense, but they represent a role of the design parameter $\psi^{s}$, and the function $\psi^{s}$ can be used to design a trade-off between accuracy of monitoring and amount of consuming energy.

One possibly simple and straightforward choice of $\psi^{s}$ is the identity map, and we set $\psi^{s}\left(\phi\left(t, v_{i}\right)\right)=\phi\left(t, v_{i}\right)$ and try to assign the activation probability as $p_{i}^{s}(t) \approx \psi^{s}\left(\phi\left(t, v_{i}\right)\right)=$ $\phi\left(t, v_{i}\right)$. Since a value of the unknown potential function $\phi\left(t, v_{i}\right)$ can not be directory used, we may need to replace it by the observed data $z_{i}(t)$ in (1) or a processed data, which are provided by the neighboring sensor nodes. In our proposed activation probability assignment algorithm in the following section, we regard that, for each node $P_{i}$, realizing $p_{i}^{s} \approx \psi^{s}$ is an ideal solution to our problem.

\section{ACTIVATION PROBABILITY ASSIGNMENT STRATEGY}

This section considers a specific activation probability assignment algorithm.

\subsection{Initial Activation Probability}

Before considering an activation probability assignment procedure, we need to investigate how to initialize the activation probability at time $t=0$.

Preceding the actual activation of the sensor network, say at time $t=-1$, we could turn on all of the sensor nodes and obtain $z_{i}(-1)=\phi\left(-1, v_{i}\right)$ for all $i \in N$. Then we could set $p_{i}^{s}(0)=\psi^{s}\left(z_{i}(-1)\right)=\psi^{s}\left(\phi\left(-1, v_{i}\right)\right)$ for all $i \in N$. This procedure requires once turn on every sensor node and may consume a certain amount of energy. If one does not desire this loss of energy, we could set $p_{i}^{s}(0) \in[0,1]$ as a random variable for each $i \in N$.

\subsection{Information from the Neighboring Sensor Nodes}

This section considers how to utilize information from the neighboring sensor nodes. We first define

$$
A_{i}(t)=\left\{j \in N_{i} \mid s_{j}(t)=1\right\} \quad t \in \mathbb{Z}^{+} \quad i \in N
$$

The set $A_{i}(t)$ consists of indices of the neighbors who are in active sensing state $\left(s_{j}(t)=1\right)$ at time $t$. We define the 
mean value $w_{i}(t), t \in \mathbb{Z}^{+}, i \in N$ of available data in the following manner.

$$
w_{i}(t)= \begin{cases}m_{i}(t)=\sum_{j \in A_{i}(t)} \frac{z_{j}(t)}{\left|A_{i}(t)\right|} & \left|A_{i}(t)\right| \neq 0 \\ p_{i}^{s}(t) & \left|A_{i}(t)\right|=0\end{cases}
$$

In the case of $\left|A_{i}(t)\right| \neq 0, w_{i}(t)$ is simply defined as the mean of all available data. On the other hand, in the case of $\left|A_{i}(t)\right|=0$, there is no active sensor node including $P_{i}$ itself and we could obtain no meaningful data, thus we define $w_{i}(t)$ as it equals to the current activation probability $p_{i}^{s}(t)$.

\subsection{Activation Probability Assignment Strategy}

We consider an activation probability assignment algorithm in Table 1 . In the algorithm, each sensor node determines its activation probability $p_{i}^{s}$ in a distributed fashion by utilizing data provided only from its neighbors.

Table 1. Probability assignment strategy.

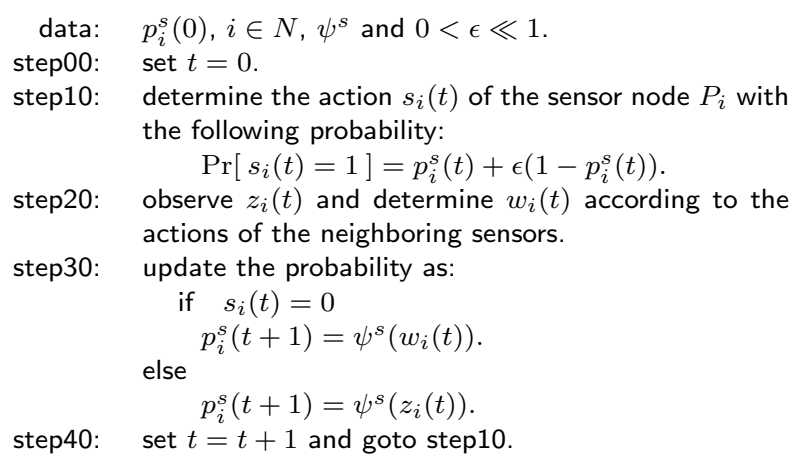

In step10, each sensor node decides its action $s_{i}(t)$ according to the assigned activation probability, where we use $p_{i}^{s}(t)+\epsilon\left(1-p_{i}^{s}(t)\right)$ instead of $p_{i}^{s}(t)$ itself. Since $\epsilon \ll 1$, the actual activation probability $p_{i}^{s}(t)+\epsilon\left(1-p_{i}^{s}(t)\right)$ is almost equal to $p_{i}^{s}(t)$, but the term $\epsilon\left(1-p_{i}^{s}(t)\right)$ realizes force flip of action $s_{i}(t)$ with very low probability defined by $\epsilon \ll 1$. This strategic flip of the activation probability according to a given parameter $0<\epsilon \ll 1$ is necessary to detect a sudden generation of the potential possibility that slope failure take place. Effectiveness of this strategic flip can be seen in a numerical example in Sections 4.4 .2 and 5.3. Each sensor node can get $z_{i}(t)$ and determine $w_{i}(t)$ in step20, since the actions $s_{i}(t), i \in N$ are already decided in step10. In the later part of step30, the node $P_{i}$ updates its activation probability as $p_{i}(t+1)=\psi^{s}\left(z_{i}(t)\right)$, when the node $P_{i}$ turns on $\left(s_{i}(t)=1\right)$ its sensing capability at time $t$ and reliable data $z_{i}(t)=\phi\left(t, v_{i}\right)$ is available as (1). On the other hand, if its sensing capability is turned off $\left(s_{i}(t)=0\right)$ at time $t$, the node $P_{i}$ uses the mean value $w_{i}(t)$ to update the activation probability.

\subsection{Numerical Experiments}

Two examples consider the activation probability assignment algorithm in Section 4.3.

Numerical Example 1 Let us consider again the deployment configuration of the sensor nodes and communication topology in Example 1. We use the activation probability assignment algorithm in Section 4.3 and set $\psi^{s}$ as the identity map and $\epsilon=0.005$.

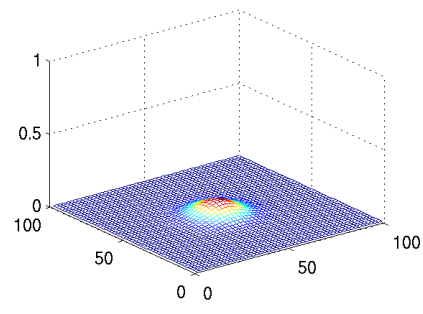

(a) $\phi(50, v), v \in S$

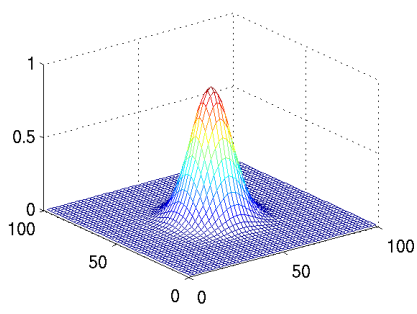

(c) $\phi(250, v), v \in S$

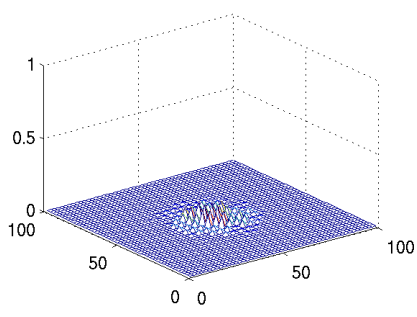

(e) $p_{i}^{s}(50), i \in N$

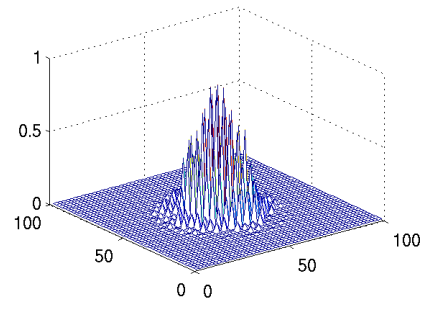

(g) $p_{i}^{s}(250), i \in N$

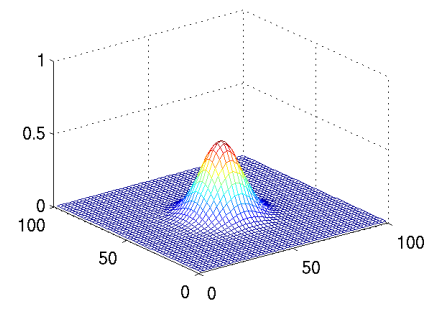

(b) $\phi(150, v), v \in S$

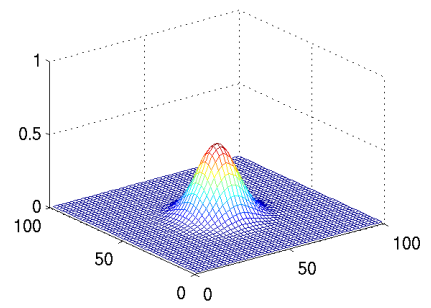

(d) $\phi(350, v), v \in S$

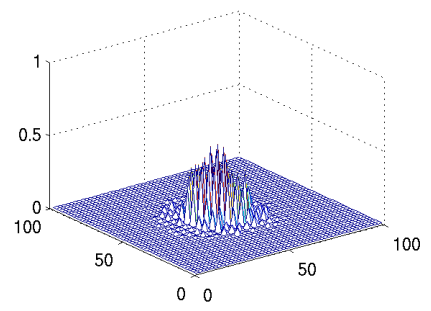

(f) $p_{i}^{s}(150), i \in N$

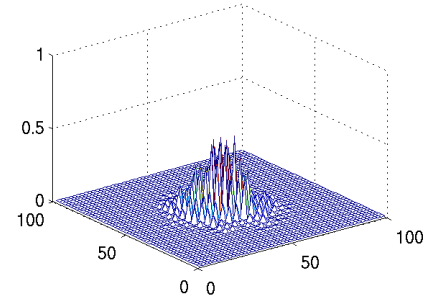

(h) $p_{i}^{s}(350), i \in N$
Fig. 3. Time evolution of the unknown potential function $\phi(\cdot, v), v \in S$ and assigned activation probability $p_{i}^{s}(\cdot), i \in N$ with identity map $\psi^{s}$ and $\epsilon=0.005$.

Snapshots of the time evolution of the unknown potential function $\phi(t, v), v \in S$ and the assigned activation probability $p_{i}^{s}(t), i \in N$ for times $t=50,150,250$ and 350 are shown in Fig. 3, respectively. We can see that the assigned activation probability $p_{i}^{s}(\cdot), i \in N$ successfully tracks to the unknown potential function $\phi(\cdot, v)=\psi^{s}(\phi(\cdot, v))$, $v \in S$.

Fig. 4 shows the action $s_{i}(t)$ of each node at times $t=$ 150 and 250. Each dot indicates the active sensing state $\left(s_{i}(t)=1\right)$. We can see an active sensing state at around $v_{i}=(44,4)$ in Fig. 4(a), due to the force flip according to the parameter $\epsilon \ll 1$.

Numerical Example 2 In this example, we set $\epsilon=0$ with the algorithm in Table 1 . Thus, the algorithm does 


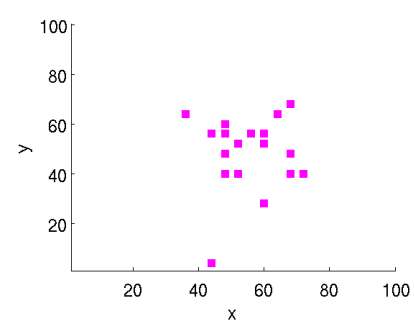

(a) $s_{i}(150), i \in N$

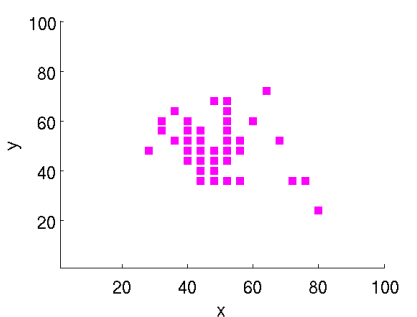

(b) $s_{i}(250), i \in N$

Fig. 4. Actions $s_{i}(\cdot), i \in N$ of the sensor nodes with identity map $\psi^{s}$ and $\epsilon=0.005$.

not introduce the force flip into the activation probability in step10, and this example may clarify usefulness of the parameter $0<\epsilon \ll 1$. The sensor nodes deployment configuration and communication topology are same to Example 1, and we use the identity map as the function $\psi^{s}$.

Snapshots of the time evolution of the unknown potential function $\phi(t, v), v \in S$ are shown in Figs. 5(a), 5(b), $5(\mathrm{c})$ and $5(\mathrm{~d})$. In this evolution of $\phi(\cdot, v), v \in S$, the large and small rectangles do not touch and are separated each other. The corresponding evolution of the assigned activation probability $p_{i}^{s}(\cdot), i \in N$ in Figs. $5(\mathrm{e}), 5(\mathrm{f}), 5(\mathrm{~g})$ and $5(\mathrm{~h})$ tracks to the evolution of large rectangle, but it can not detect the generation of small rectangle. Since the two rectangles are separated and the communication topology allows to interact with the left, right, top and bottom sensors only, a positive activation probability over the region of large rectangle can not propagate to the region of small rectangle being generate. If the communication topology allowed each node to interact with other nodes having more distance, the algorithm successfully catches up the sudden generation of the small rectangle. In addition, if we set as $\epsilon>0$, the algorithm also successfully tracks to this unknown potential function as the numerical example in Section 5.3.

\section{SENSING AND COMMUNICATION ACTIVATION PROBABILITY}

This section considers a generalized problem that involves activation or deactivation of both sensing and communication capabilities.

\subsection{Activation Probability Assignment}

The actual sensor node in practice has sensing and communication capabilities. For each $P_{i}, i \in N$, we assign another action set $A_{i}^{c}=\{0,1\}$, which represents an activity of communication capability. The action $c_{i}(t)=1 \in A_{i}^{c}$ implies that the node $P_{i}$ activates its communication channel. We denote the entire action of each node $P_{i}$ as $a_{i}(t)=\left(s_{i}(t), c_{i}(t)\right) \in A_{i}^{s} \times A_{i}^{c}=\{0,1\}^{2}$. As similar to the sensing activation problem, let $p_{i}^{c}(t) \in[0,1], t \in \mathbb{Z}^{+}, i \in$ $N$ denote the communication activation probability. The node $P_{i}$ activate its communication capability according to the assigned probability, i.e., $\operatorname{Pr}\left[c_{i}(t)=1\right]=p_{i}^{c}(t)$. The function $\psi^{s}$ in the sensing capability activation problem can be regarded as a function measuring the importance of sensing activity. As similar to this, we introduce a function

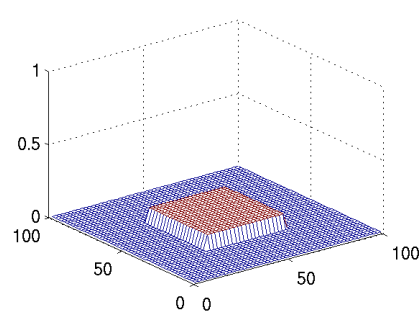

(a) $\phi(50, v), v \in S$

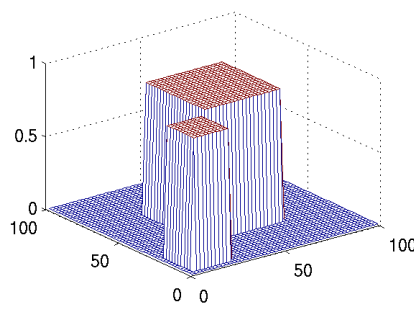

(c) $\phi(250, v), v \in S$

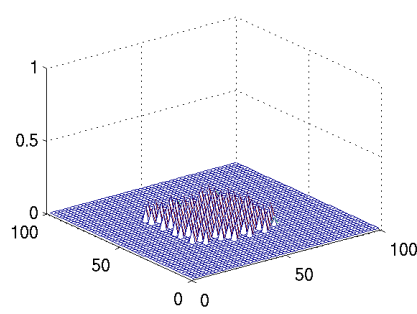

(e) $p_{i}^{s}(50), i \in N$

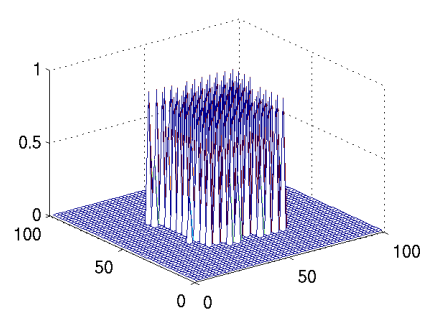

(g) $p_{i}^{s}(250), i \in N$
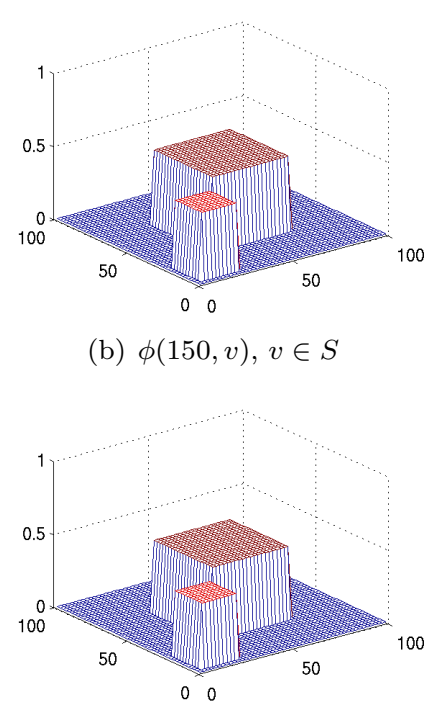

(d) $\phi(350, v), v \in S$

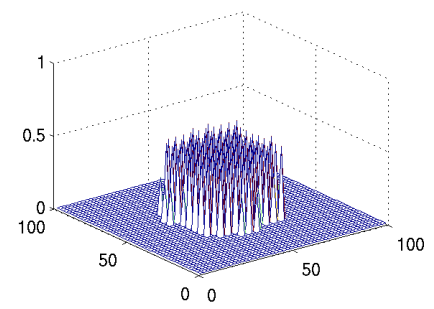

(f) $p_{i}^{s}(150), i \in N$

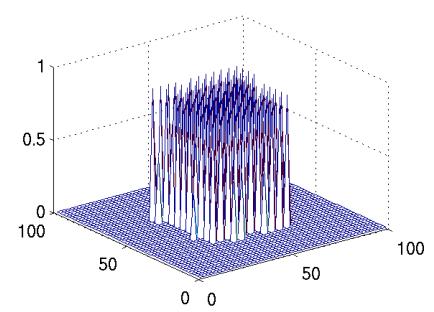

(h) $p_{i}^{s}(350), i \in N$ (b) $\phi(150, v), v \in S$

Fig. 5. Time evolution of the unknown potential function $\phi(\cdot, v), v \in S$ and assigned activation probability $p_{i}^{s}(\cdot), i \in N$ with identity map $\psi^{s}$ and $\epsilon=0$. By setting to $\epsilon>0$, the algorithm can successfully track to this type of unknown potential function (see numerical example in Section 5.3).

$\psi^{c}$ that will represent an importance of interactions with the neighboring nodes. We consider the following examples.

Example 2. Let us suppose that, as we have considered thus far, the targeted sensing activation probability assignment $p_{i}^{s}(t)=\psi^{s}\left(\phi\left(t, v_{i}\right)\right)$ is realized. In the problem of slope disaster detection considered here, in case of $\phi\left(\cdot, v_{i}\right) \approx 1$, the nodes at a neighboring location of $v_{i}$ may have small incentive to turn on its communication capability, since the node who turn on its sensing capability $\left(s_{i}(t)=1\right)$ according to the assigned sensing activation probability $p_{i}^{s}(\cdot)=\psi^{s}\left(\phi\left(\cdot, v_{i}\right)\right) \approx \psi^{s}(1)$ can obtain a reliable data as in (1) and may not need to interact neighbors to receive data. Similarly, in case of $\phi\left(\cdot, v_{i}\right) \approx 0$, the nodes may also have small incentive to turn on the 


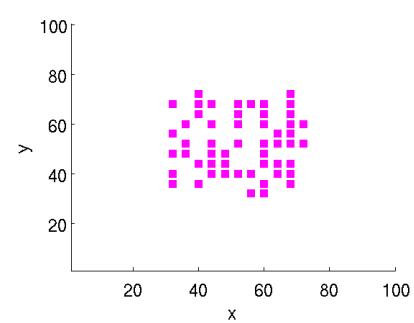

(a) $s_{i}(150), i \in N$

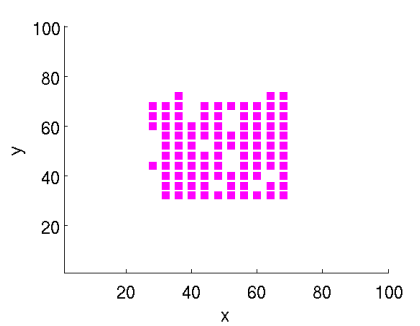

(b) $s_{i}(250), i \in N$
Fig. 6. Actions $s_{i}(\cdot), i \in N$ of the sensor nodes with identity map $\psi^{s}$ and $\epsilon=0$.

communication capability, because there is a small potential possibility that the slope failure take place. On the other hand, if the value of $\phi\left(\cdot, v_{i}\right)$ takes medium, only a half the number of nodes turn on its sensing capability, and observed data is scarce. Therefore, in order to share scarce data, the interactions between neighboring nodes become more important. One can consider the following candidates of the function $\psi^{c}$ that represents the above situations.

$$
\begin{aligned}
& \psi^{c}(q(t, s))=4 \times q(t, s) \times(1-q(t, s)) \\
& \psi^{c}(q(t, s))= \begin{cases}2 \times q(t, s) & 0 \leq q(\cdot, \cdot)<1 / 2 \\
-2 \times q(t, s)+2 & 1 / 2 \leq q(\cdot, \cdot) \leq 1\end{cases}
\end{aligned}
$$

If we set $q(t, v)=\phi(t, v)$ and consider the case $\phi(t, v)=1$ or 0 , the both $\psi^{c}$ in (5) and (4) become 0 . If the value of $\phi(t, v)$ is $1 / 2$, then we have $\psi^{c}=1$. Therefore, if the targeted communication activation probability assignment $p_{i}^{c}(t)=\psi^{c}\left(\phi\left(t, v_{i}\right)\right)$ was realized, we have dense interactions between the nodes in case of $\phi(t, v)=1 / 2$.

Example 3. In contrast to Example 2, one may find importance on the interactions between the nodes for the case of $\phi(\cdot, \cdot) \geq 1 / 2$. The function

$$
\psi^{c}(q(t, s))= \begin{cases}2 \times q(t, s) & 0 \leq q(\cdot, \cdot)<1 / 2 \\ 1 & 1 / 2 \leq q(\cdot, \cdot) \leq 1\end{cases}
$$

can be a possible candidate of $\psi^{c}$ in this case.

The function $\psi^{c}$ is a design parameter in our problem and can be used to represent importance of interactions between the nodes. In the following probability assignment algorithm, we regard that, for each node $P_{i}$, realizing $p_{i}^{s} \approx \psi^{s}$ and $p_{i}^{c} \approx \psi^{c}$ is an ideal solution to our problem.

\subsection{Activation Probability Assignment Strategy}

We need to start with modifying the definition of the set of active neighbors in (2). Let us define

$$
A_{i}(t)=\left\{j \in N_{i} \mid s_{j}(t) \times c_{j}(t)=1\right\} \quad t \in \mathbb{Z}^{+} \quad i \in N
$$

The set $A_{i}(t)$ consist of indices of the neighbors who are in active both sensing and communication states $\left(a_{i}(t)=\right.$ $\left.\left(s_{i}, c_{i}(t)\right)=(1,1)\right)$ at time $t$. The mean value of available data in (3) is redefined as

$w_{i}(t)= \begin{cases}m_{i}(t)=\sum_{j \in A_{i}(t)} \frac{z_{j}(t)}{\left|A_{i}(t)\right|} & c_{i}(t)=1 \text { and }\left|A_{i}(t)\right| \neq 0 \\ p_{i}^{s}(t) & \text { otherwise }\end{cases}$

If the node $P_{i}$ activated its communication channel and had at least one active neighbor, $w_{i}(t)$ is simply defined as the mean of all available data. Otherwise, including the case that the node $P_{i}$ deactivated its communication channel, we define $w_{i}(t)$ as it equals to the current sensing activation probability $p_{i}^{s}(t)$.

A sensing and communication activation probability assignment strategy, which generalizes the algorithm in Table 1, can be proposed as in Table 2 .

Table 2. Sensing and communication activation probability assignment strategy.

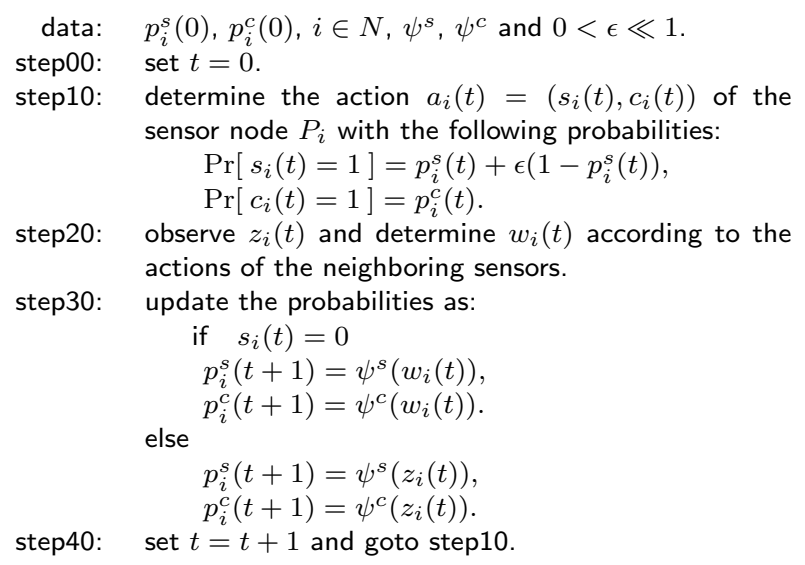

In the algorithm in Tables 2, we do not introduce a flip of the communication activation probability, while we use it for the sensing activation according to the given parameter $0<\epsilon \ll 1$. The sensing capability is once activated, the node obtains a reliable data as in (1), and the communication activation probability is updated with the reliable data as $p_{i}^{c}(t+1)=\psi^{c}\left(z_{i}(t)\right)=\psi^{c}\left(\phi\left(t, v_{i}\right)\right)$. Actually, throughout our numerical experiment studies, we confirmed that the time evolution of assigned activation probability successfully tracks to the time evolution of the unknown potential function, and we conclude that a flip of the communication activation probability is not necessarily needed.

We would like to close this subsection with the following remark. This Section 5 considers turn on/off strategies of the communication capability of each node from energy consumption point of view only. Each node not only needs to send a data, but also needs to receive and forward data from its neighbors. We need to keep a communication path from a specific node to the base station, especially when this specific node generates an alert signal in case of emergence. Thus, the problem of activating or deactivating the communication capability should be solved under the path keeping constraints Shi et al. (2008). For a real practice purpose, the communication capability activation probability assignment problem in this section may need to have a further investigation under this path keeping constraints.

\subsection{Numerical Experiment}

Let us consider again the deployment configuration of the sensor nodes and communication topology in Example 1. We use the sensing and communication activation probability assignment strategy in Table. 2 and set $\psi^{s}$ as the identity map, a positive number $\epsilon$ as 0.005 and the function $\psi^{c}$ as in (4). 


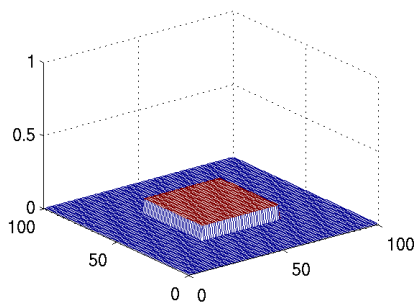

(a) $\phi(50, v), v \in S$

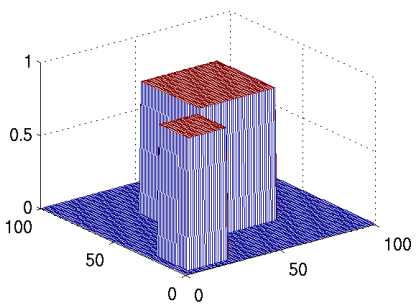

(c) $\phi(250, v), v \in S$

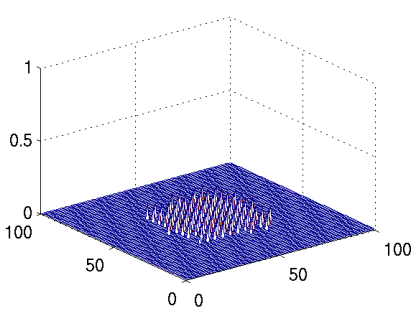

(e) $p_{i}^{s}(50), i \in N$

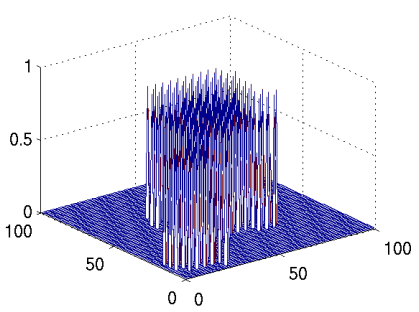

(g) $p_{i}^{s}(250), i \in N$

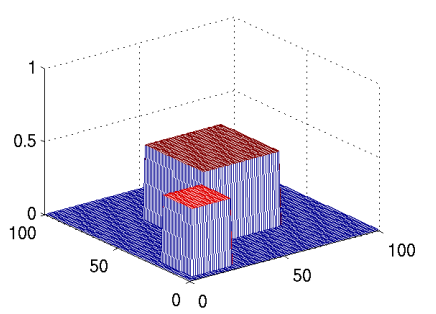

(b) $\phi(150, v), v \in S$

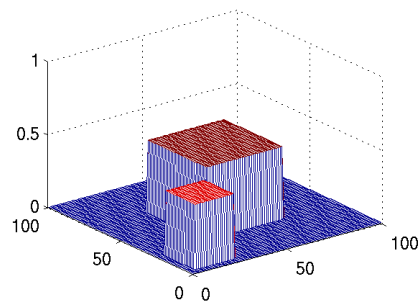

(d) $\phi(350, v), v \in S$

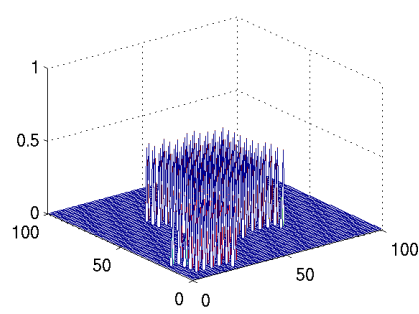

(f) $p_{i}^{s}(150), i \in N$

(h) $p_{i}^{s}(350), i \in N$

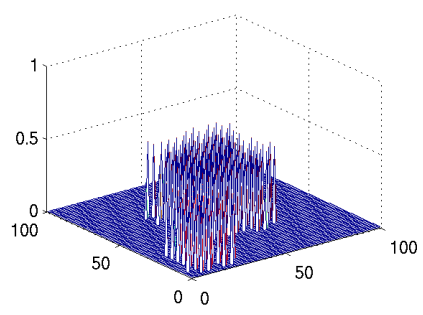

Fig. 7. Time evolution of the unknown potential function $\phi(\cdot, v), v \in S$ and assigned activation probability $p_{i}^{s}(\cdot), i \in N$ with identity map $\psi^{s}$, function $\psi^{c}$ in (4) and $\epsilon=0.005$. We omit the figures of time evolution of $p_{i}^{c}(\cdot), i \in N$, but it successfully tracks to the time evolution of $\psi^{c}(\phi(\cdot, v)), v \in S$. A positive $\epsilon>0$ makes possible to detect a sudden generation of small rectangle (see and compare numerical example in Section 4.4.2).

Snapshots of the time evolution of the unknown potential function $\phi(t, v), v \in S$ and the assigned sensing activation probability $p_{i}^{s}(t), i \in N$ for times $t=50,150,250$ and 350 are shown in Fig. 7, respectively. Although, in contrast to the results in Figs 3 and 5, this example only uses non-dense communication interactions between the nodes that is decided according to the assigned communication activation probability $p_{i}^{c}(\cdot), i \in N$, we can see that the assigned sensing activation probability $p_{i}^{s}(\cdot), i \in N$ successfully tracks to the unknown potential function $\phi(\cdot, v)=\psi^{s}(\phi(\cdot, v)), v \in S$. We omit the figures of time evolution of $p_{i}^{c}(\cdot), i \in N$, but it also successfully tracks to the time evolution of $\psi^{c}(\phi(\cdot, v)), v \in S$.

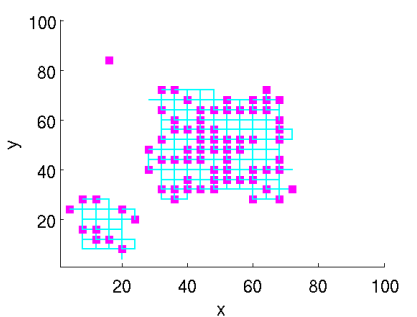

(a) $s_{i}(150)$ and $c_{i}(150), i \in N$

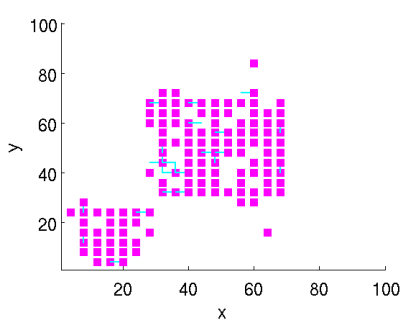

(b) $s_{i}(250)$ and $c_{i}(250), i \in N$
Fig. 8. Actions $a_{i}(\cdot)=\left(s_{i}(\cdot), c_{i}(\cdot)\right), i \in N$ of the sensor nodes with identity map $\psi^{s}$, function $\psi^{c}$ in (4) and $\epsilon=0.005$.

Fig. 8 shows the action $a_{i}(t)=\left(s_{i}(t), c_{i}(t)\right)$ of each node at times $t=150$ and 250. Each dot indicates the active sensing state $\left(s_{i}(t)=1\right)$, and each edge indicates the active communication state $\left(c_{i}(t)=1\right.$ and $c_{j}(t)=1$ where $\left.j \in N_{i}\right)$. At time $t=250$, due to a high sensing activation probability $p_{i}^{s}(250) \approx \psi^{s}\left(\phi\left(250, v_{i}\right)\right)=\phi\left(250, v_{i}\right)=0.9$, almost all the sensing capabilities are activated. On the other hand, $p_{i}^{c}(150) \approx \psi^{c}\left(\phi\left(150, v_{i}\right)\right)$ and $\psi^{c}\left(\phi\left(150, v_{i}\right)\right)=$ $\psi^{c}(0.5)=1$ establish a dense interactions between the nodes at time $t=150$.

\section{CONVERGENCE ANALYSIS}

This section investigates convergence property of the proposed algorithm. We apply the standard Lyapunov argument and will see that the Lyapunov function defined for each $P_{i}$ is ultimately bounded. For the sake of simplicity of notations, we consider the algorithm in Table 1 with the identity map $\psi^{s}$, but the extension to the case of both sensing and communication probability assignment with the algorithm in Table 2 is straightforward.

Let us suppose that there exists $t_{0} \geq 0$ such that the unknown time-varying function $\phi(t, v)$ converges to some constant value for all $t \geq t_{0}$ and all $v \in S$. For each $i \in N$, we set

$$
\phi\left(t, v_{i}\right)=\phi_{i} \quad \text { for all } t \geq t_{0}
$$

Theorem 1. For each $i \in N$, let us define $V_{i}(t)=\left(p_{i}^{s}(t)-\right.$ $\left.\phi_{i}\right)^{2}$ and $\mathrm{E}[\cdot]$ denote the expectation. We have

$\lim _{t \rightarrow \infty} \mathrm{E}\left[V_{i}(t) \mid p_{j}^{s}(t-1), j \in N_{i}\right] \leq \frac{1-\epsilon}{\epsilon} \max _{k \in N_{-i}}\left(\phi_{k}-\phi_{i}\right)^{2}$ where $N_{-i}=N_{i} \backslash\{i\}$.

Let us set $u_{i}=\max _{k \in N_{-i}}\left(\phi_{k}-\phi_{i}\right)^{2}$, and it evaluates the largest difference between the values of unknown potential function over the neighbors in $N_{i}$. Theorem 1 concludes that if the variation of unknown potential function $\phi$ was moderately small or the sensor nodes ware densely deployed, the expectation of $\left(p_{i}^{s}(t)-\phi_{i}\right)^{2}$ converges to a sufficiently small value.

The remaining of this section proves Theorem 1 . For each $P_{i}, i \in N$, let $p_{j}^{s}(t), j \in N_{i}, t \geq t_{0}$ be given sensing activation probability of its neighbors. According to the algorithm in Table 1, we have the evolution of $p_{i}^{s}$ as 


$$
p_{i}^{s}(t+1)=\left\{\begin{array}{rr}
\phi_{i} \quad \text { with probability } p_{i}^{s}(t)+\epsilon\left(1-p_{i}^{s}(t)\right) \\
m_{i}(t) \quad \text { with probability } \\
(1-\epsilon)\left(\left(1-p_{i}^{s}(t)\right)-\prod_{j \in N_{i}}\left(1-p_{j}^{s}(t)\right)\right) \\
p_{i}^{s}(t) \quad \text { with probability } \\
\quad(1-\epsilon) \prod_{j \in N_{i}}\left(1-p_{j}^{s}(t)\right)
\end{array}\right.
$$

Let us consider a candidate Lyapunov function $V_{i}(t)=$ $\left(p_{i}^{s}(t)-\phi_{i}\right)^{2}$, and, by substituting $p_{i}^{s}(t+1)$, we have that $\mathrm{E}\left[V_{i}(t+1)-V_{i}(t) \mid p_{j}^{s}(t), j \in N_{i}\right]$

$$
\begin{aligned}
= & \left(m_{i}(t)-\phi_{i}\right)^{2} \times(1-\epsilon)\left(\left(1-p_{i}^{s}(t)\right)-\prod_{j \in N_{i}}\left(1-p_{j}^{s}(t)\right)\right) \\
& +\left(p_{i}^{s}(t)-\phi_{i}\right)^{2} \times(1-\epsilon) \prod_{j \in N_{i}}\left(1-p_{j}^{s}(t)\right)-V_{i}(t) \\
\leq & \left(m_{i}(t)-\phi_{i}\right)^{2} \\
& \times(1-\epsilon)\left(\left(1-p_{i}^{s}(t)\right)-\prod_{j \in N_{i}}\left(1-p_{j}^{s}(t)\right)\right)-\epsilon V_{i}(t)
\end{aligned}
$$

where we use $-1+(1-\epsilon) \prod_{j \in N_{i}}\left(1-p_{j}^{s}(t)\right) \leq-\epsilon$. From the definition of $u_{i}$ and $\left(1-p_{i}(t)\right)-\prod_{j \in N_{i}}\left(1-p_{j}(t)\right)=(1-$ $\left.p_{i}(t)\right)\left(1-\prod_{j \in N_{-i}}\left(1-p_{j}(t)\right)\right)<1$, we further have

$$
\mathrm{E}\left[V_{i}(t+1) \mid p_{j}(t), j \in N_{i}\right] \leq(1-\epsilon) V_{i}(t)+(1-\epsilon) u_{i}
$$

According to the standard comparison principle for discrete-time systems Sugiyama (1970), we have

$$
\mathrm{E}\left[V_{i}(t) \mid p_{j}(t-1), j \in N_{i}\right] \leq x_{i}(t) \quad t \geq t_{0}
$$

where $x_{i}(t)$ is the solution to the initial value problem

$$
x_{i}(t+1)=(1-\epsilon) x_{i}(t)+(1-\epsilon) u_{i} \quad x\left(t_{0}\right)=V_{i}\left(t_{0}\right)
$$

Thus, we have

$$
\begin{aligned}
\mathrm{E}\left[V_{i}(t) \mid p_{j}(t-1), j\right. & \left.\in N_{i}\right] \leq(1-\epsilon)^{t-t_{0}} V_{i}\left(t_{0}\right) \\
& +\sum_{k=t_{0}}^{t-1}(1-\epsilon)^{\left(k-t_{0}+1\right)} u_{i}
\end{aligned}
$$

Since $(1-\epsilon)<1$, by letting $t \rightarrow \infty$, we have $\sum_{k=t_{0}}^{t-1}(1-$ $\epsilon)^{\left(k-t_{0}+1\right)}=\lim _{t \rightarrow \infty} \sum_{k=0}^{t-1-t_{0}}(1-\epsilon)^{(k+1)}=(1-\epsilon) / \epsilon$, and this concludes the theorem.

\section{CONCLUSIONS}

This paper addressed a design of operating function of a distributed sensor network for slope disaster detection. We considered a low energy consumption property of the entire sensor network and proposed a probabilistic turn on/off strategy of each sensor node. The proposed algorithm assigns the activation probability for each sensor node according to the observed slope failure index, and this probability assignment strategy can be realized in a distributed fashion. Effectiveness of the proposed strategy was evaluated through numerical experiments, and the results showed that a resulting assigned activation probability can track a time evolution of the unknown time-varying slope failure potential index. This paper also investigated convergence property of the proposed algorithm. We applied the Lyapunov argument and showed the ultimate boundedness of the Lyapunov function. An experimental evaluation of the proposed algorithm using hardware devices have been reported and can be found in Hirata et al. (2013).

\section{REFERENCES}

Chigira, M. (2006). Prediction of potential landslide sites -From the viewpoint of geology and geomorphology-. Journal of Japan Society of Civil Engineers, Ser. C, 62(4), 722-735. In Japanese.

Fukagawa, R. et al. (2008). Resarch on a sensor network system for slope failure due to rainfall. In Peoceeding of the Symposium of Jiban-no-Kankyou-Keisokugijutu, 21-26. In Japanese.

Gharavi, H. and Kumar, S.P. (2003). Special issue on sensor networks and applications. Proceedings of the IEEE, 91(8).

Gupta, V., Chung, T.H., Hassibi, B., and Murray, R.M. (2006). On a stochastic sensor selection algorithm with applications in sensor scheduling and sensor coverage. Automatica, 42(2), $251-260$.

Hespanha, J.P., , Naghshtabrizi, P., and Xu, Y. (2007). A survey of recent results in networked control systems. Proceedings of the IEEE, 95(1), 138-162.

Hirata, K., Koizumi, K., and Yoshitake, M. (2013). Probabilistic management of slope disaster detection systems for reduced energy consumption. In Proceedings of the 2013 American Control Conference, 5048/5055. Washington, DC.

Ikegawa, Y. et al. (2009). Monitoring of an active slope by wireless sensor network. In Proceedings of the 38th Symposium on Rock Mechanics, 39-44. In Japanese.

Jia, Q.S., Shi, L., Mo, Y., and Sinopoli, B. (2012). On optimal partial broadcasting of wireless sensor networks for kalman filtering. IEEE Transactions on Automatic Control, 57(3), 715 -721.

Kim, K.T. and Han, J.G. (2008). Design and implementation of a real-time slope monitoring system based on ubiquitous sensor network. In Proceedings of the 25th International Symposium on Automation and Robotics in Construction, 330-336.

Kitamura, R. (2007). Monitoring technique of slope stability in the rain. Tsuchi-to-Kiso, 55(9), 1-3. In Japanese.

Koizumi, K. et al. (2011). Slope disaster monitoring system using battery-operated wireless sensor network. In The 2nd World Landslide Forum, WLF-0263. Rome, Italy.

Koizumi, K. et al. (2012a). Slope disaster detection system using sensor networks and its field experiment evaluations. SICE Journal of Control Measurement, and System Integration, 5(1), $41-47$.

Koizumi, K. et al. (2012b). Slope disaster monitoring system using wireless mesh-type network. In Proceedings of the International Workshop on ICT in Geo-Engineeting 2012, 107-115. Kyoto, Japan.

Mehta, P. et al. (2007). Distributed detection for landslide prediction using wireless sensor network. In First International Global Information Infrastructure Symposium, 195-198.

Miller, M. and Vaidya, N. (2005). A MAC protocol to reduce sensor network energy consumption using a wakeup radio. IEEE Transactions on Mobile Computing, 4(3), 228 - 242.

Mo, Y., Ambrosino, R., and Sinopoli, B. (2011). Sensor selection strategies for state estimation in energy constrained wireless sensor networks. Automatica, 47(7), $1330-1338$.

Nishiyama, S. et al. (2008). Study on real-time rock slope monitoring network system. In Proceedings of the 37th Symposium on Rock Mechanics, 439-442. In Japanese.

Ramesh, M.V. (2009). Real-time wireless sensor network for landslide detection. In Third International Conference on Sensor Technologies and Applications, 405-409.

Schwab, A.K., Eschelbach, K., and Brower, D.J. (2007). Hazard Mitigation and Preparedness. Wiley.

Shi, L., , A.C., Johansson, K.H., and Murray, R.M. (2008). Sensor network lifetime maximization via sensor trees construction and scheduling. In Third International Workshop on Feedback Control Implementation and Design in Computing Systems and Networks.

Sugiyama, S. (1970). Comparison theorems on difference equations. Bull. Sci. Eng. Res. Lab., Waseda University., 47(77-82).

Terzis, A. et al. (2006). Slip surface localization in wireless sensor networks for landslide prediction. In Proceedings of the 5th international conference on Information processing in sensor networks, 109-116. 